\title{
Direct and indirect effects of parent stress on child obesity risk and added sugar intake in a sample of Southern California adolescents
}

\author{
Eleanor T Shonkoff ${ }^{1, *}$, Genevieve F Dunton ${ }^{2}$, Chih-Ping Chou ${ }^{2}$, Adam M Leventhal ${ }^{2}$, \\ Ricky Bluthenthal ${ }^{2}$ and Mary Ann Pentz ${ }^{2}$ \\ ${ }^{1}$ Tufts University, Child Obesity 180, Tufts University Friedman School of Nutrition Science and Policy, 150 Harrison \\ Avenue, Boston, MA 02111 , USA: ${ }^{2}$ University of Southern California, Department of Preventive Medicine, \\ Los Angeles, CA, USA
}

Submitted 4 April 2017: Final revision received 3 August 2017: Accepted 7 August 2017: First published online 5 0ctober 2017

\begin{abstract}
Objective: Research indicates that children are at higher risk for obesity if their parents have been exposed to a larger number of stressors, yet little is known about effects of parents' subjective, perceived experience of stress on children's eating behaviours and adiposity and whether weight-related parenting practices (i.e. parent rules and positive family meal practices) mediate this relationship. The present study evaluated the direct and mediated relationship between parent perceived stress and child waist circumference and parent stress and child consumption of added sugars one year later.

Design: Longitudinal panel data.

Setting: Eleven communities in Southern California, USA.

Subjects: Data were collected over two waves from parent-child dyads ( $n$ 599). Most parents were female (81\%) and Hispanic (51\%); children were 11 years old on average (SD 1.53; range 7-15 years) and $31 \%$ received free school lunch.

Results: Perceived parent stress was not significantly associated with child waist circumference or consumption of added sugars one year later, and mediating pathways through parenting practices were not significant. However, parent rules were significantly associated with lower child consumption of added sugars $(\beta=-0 \cdot 14, P<0 \cdot 001)$.

Conclusions: Results suggest that parent rules about the types of foods children can eat, clearly explained to children, may decrease child consumption of added sugars but not necessarily lead to changes in obesity risk. Parent- and family-based interventions that support development of healthy rules about child eating have the potential to improve child dietary nutrient intake.
\end{abstract}

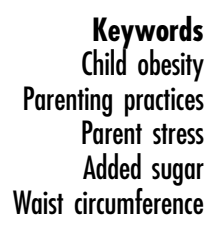

Parent stress (e.g. food insecurity, parenting stress, distress, maternal stressors and financial strain) has been linked to higher child obesity risk (e.g. ${ }^{(1-7)}$ ). Possibly, stress changes parents' behaviour in ways that negatively impact children's weight-related behaviour, such as lowering consumption of fruits and vegetables or increasing sedentary behaviour ${ }^{(8,9)}$. According to an adapted Family Stress Model perspective, poverty (a significant stressor) could lead to parental depression, disrupting the interparent relationship, altering parenting behaviours such as beneficial feeding practices, and ultimately heightening child obesity risk ${ }^{(10,11)}$. Poverty and other stressors would likely lead to perceived stress as well, having similar downstream effects on the interparent relationship, a parent's ability to maintain and enforce healthy parenting practices, and an increase in unhealthy child dietary intake and obesity risk. Thus, conceptually, the effects of 'objectively' stressful parent circumstances (i.e. stressors) on child obesity risk would be expected to mirror those of subjective perceived parent stress, which has been defined as the psychological experience of evaluating oneself as unable to meet the demands of an event or circumstance $^{(12)}$. However, environmental 'objective' stressors, perceived stress and biological stress responses may occur at different points along a stress process ${ }^{(12)}$ and could have different impacts on child obesity risk ${ }^{(10,11)}$.

Little previous research has assessed unique effects of parent perceived stress separately from stressors and these studies have been limited by cross-sectional study designs. One study found that parents' perceived stress was 
associated with $7 \%$ higher odds of obesity in children aged 3-17 years, but the relationship became nonsignificant after adjusting for physical and environmental stressors (i.e. physical health, financial strain, family structure), psychological well-being (i.e. mental health) and other covariates ${ }^{(13)}$. Another found that higher parenting stress (a specific type of perceived stress associated with the responsibilities of parenting) was not associated with child risk of obesity ${ }^{(14)}$. Yet it remains unknown whether an investigation over a longer time period would find similar results - parent stress could disrupt parenting practices in ways that accumulate over time, eventually impacting child obesity risk.

Parent perceived stress has been related to weightrelated parenting practices and weight-related parenting practices have been linked to child obesity risk. Both parent perceived stress and parenting stress, specifically, have been associated with child-feeding styles (e.g. uninvolved, authoritarian, restrictive), less limiting of children's television time and lower likelihood of children meeting physical activity guidelines, indicating that perceived stress may affect certain weight-related parenting practices $^{(14-16)}$. The parenting practices of having rules about child diet, having restrictive rules (e.g. snacking or kitchen access) and family meal practices (e.g. less frequent family dinner) have been connected to both more frequent child consumption of fatty foods and higher BMI percentile or $Z$-score, but also to lower likelihood of consuming sugary foods ${ }^{(17-21)}$. High consumption of added sugars, particularly from sugar-sweetened beverages, has been associated with child weight gain, obesity risk and risk for metabolic syndrome ${ }^{(22-24)}$. But whether parent rules could curb sugar intake - and possibly obesity risk - is unknown. Among adolescents and toddlers, restriction rules have been associated with lower consumption of soda and sweet foods like cookies ${ }^{(21,25)}$. But one study found that while kindergarteners whose parents had restrictive rules did consume fewer sugary beverages, children did not consume fewer total added sugars; and they preferred sweeter-tasting beverages, possibly setting the stage for higher future consumption of sugary foods ${ }^{(20)}$. Thus, longitudinal studies are critical to understanding the interplay between parenting practices, child consumption of added sugars (particularly sugarsweetened beverages) and child obesity risk. If parent perceived stress disrupts weight-related parenting practices, particularly those related to child sugar consumption, children's risk for obesity could increase.

Despite evidence for the intermediary role of parenting practices, only one known study to date has tested a statistical mediation model of parent stress leading to changes in parenting practices and subsequent child obesity. That study found that lack of family rules, time demands and difficulty enforcing rules - all of which they considered to be objective stressors - decreased positive family meal practices, leading to higher child BMI $Z$-score; but perceived parent stress did not have this indirect effect $^{(26)}$. Yet that cross-sectional study did not test effects over time and examined only one parenting practice: arrangement of family meals. Further, they conceptualized lack of parent rules as a stressor, but there may be more upstream contributing factors, such as parent perceived stress, that compromise a parent's ability to maintain and enforce rules, instead of lack of rules being stressors themselves. Finally, effects of parental stress on children's waist circumference may differ from those on BMI. Waist circumference is an indicator of abdominal obesity ${ }^{(27,28)}$ and consumption of added sugars, specifically from sugarsweetened beverages, has been associated with abdominal adiposity among children ${ }^{(29,30)}$. Taken together, these findings suggest that parent stress could contribute to child diet or obesity risk indirectly by changing relevant parenting practices, such as rules or family meal practices, but longitudinal mediated effects remain largely unstudied and mediated effects on child diet are unknown.

To address these gaps, the current study used longitudinal panel data from parent - child dyads to test a mediational model of the effects of parent perceived stress on parenting practices (i.e. parent rules and positive family meal practices) one year later and child waist circumference and added dietary sugar that year. We hypothesized that parent stress would be negatively correlated with parenting practices and positively correlated with child added sugar intake and child waist circumference (Hypothesis 1); and that higher perceived parent stress would predict: greater increases in child waist circumference and consumption of added sugars over one year (Hypothesis 2); lower parent rules about child diet and positive family practices, which in turn would predict higher child waist circumference via the mediational pathway (Hypothesis 3); and lower parent rules about child diet and positive family practices, which in turn would predict higher child consumption of added sugars via the mediational pathway (Hypothesis 4).

\section{Methods}

\section{Participants}

Participants were from a larger 5-year, matched-control trial called Healthy PLACES, investigating effects of the built environment on child obesity risk in a smart growth community ${ }^{(31,32)}$. Participants lived in the smart growth community (treatment group) or one of the matched control communities (control group). Participant families included one parent and one child aged 8-14 years. Eligibility criteria were: (i) having one child enrolled in grades 4-8; (ii) living in Chino, California, USA or surrounding communities with their child; (iii) an ability to read English; and (iv) having an annual household income $<$ \$US 210 000. Parents gave written informed consent and children gave minor assent. The Institutional Review 
Board at the University of Southern California approved the study. Participants were 610 parent - child dyads, 599 of whom provided information on relevant study measures and comprised the analytic sample.

\section{Procedures}

Recruitment procedures have been reported in detail elsewhere $^{(31-35)}$. Data were collected either at a local community site or participants' homes. Participants completed all measures on a paper survey at two time points. Measures were not adapted for the present study to allow comparability of findings across studies. Baseline data were collected between March 2009 and December 2010 (time 1; T1), during which no data collection occurred from late July through August or during January due to extreme heat and rainy weather conditions, respectively, limiting outside activity in Southern California during those months. The second wave of data collection occurred between 6 and 12 months after baseline (time 2; T2).

\section{Measures}

\section{Demographics}

Parents and children provided the following information: parent gender, child age, child ethnicity and free/reducedprice school lunch status. These demographic characteristics were included as covariates in the model for the following reasons. Evidence indicates that children's risk for obesity tends to mirror that of their same-sex parent (e.g. sons mirror fathers), suggesting the link has an environmental basis rather than genetic ${ }^{(36)}$. Child age was calculated based on the child's birth day, month and year. Older children may be less susceptible to the effects of parent stress as peers or media begin to play a more influential role in dietary choices ${ }^{(37)}$. Ethnicity was collapsed into Hispanic $v$. non-Hispanic due to a sizeable proportion of Hispanic participants in the research population. Free/reduced-price school lunch status was used as a proxy measure of socio-economic status, as income or education could affect parent stress or parenting behaviours, although findings are mixed ${ }^{(38-40)}$.

\section{Child measures}

Block Kids' Dietary Screener. The NutritionQuest Block Kids' Dietary Screener for children aged 2-17 years was used to assess child dietary intake 'over the last week' ${ }^{(41)}$. The Screener has been validated against a $24 \mathrm{~h}$ recall method for use in adolescents for whole grains and meat/ fish/poultry consumption. It is a brief measure that is useful for field-based data collection when clinical methods, such as weighed plate waste, are not available ${ }^{(42)}$. All added sugars were consistent with those in the US Department of Agriculture's My Pyramid Equivalents Database ${ }^{(43)}$, which includes sugars from condiments like ketchup. Standard adjustments to dietary variables were made; thus, total added sugar was adjusted to reflect the number of teaspoons of added sugars per $1000 \mathrm{kcal}$ consumed ((total teaspoons of added sugar/total daily $\mathrm{kcal}) \times 1000 ; 1 \mathrm{kcal}=4.184 \mathrm{~kJ})$. Screening and cleaning procedures are described in the data analysis section.

Child waist circumference. Child waist circumference was measured in duplicate using a flexible tape measure. An average of the two measures was calculated. Waist circumference has been highly correlated with BMI as an indicator of obesity but may be a better indicator of child abdominal obesity and adult metabolic syndrome ${ }^{(27,28)}$ and has been associated with consumption of added sugars such as fructose $\mathrm{e}^{(29,30)}$.

\section{Parent measures}

Parent perceived stress. The four-item version of the Cohen Perceived Stress scale was used to assess parent stress in the past $30 \mathrm{~d}^{(44)}$ (for items, see Table 1). Two items were reverse-coded and a sum of the four items was calculated to create an overall stress score, consistent with the validation study ${ }^{(44)}$. The scale has been widely used and validated ${ }^{(44-46)}$. The four-item scale has been found to have somewhat lower internal validity than the full fourteen-item scale, but it has demonstrated acceptable reliability and is recommended for use when there is a need to minimize participant burden, as there was in the current study ${ }^{(44,46)}$. Internal reliability was shown to be acceptable in this sample at baseline and follow-up (T1, $\alpha=0.73 ; \mathrm{T} 2, \alpha=0.74)^{(44)}$.

Parent rules about child eating. Three items from the Lack of Family Rules scale assessed whether parents had rules about child eating and communicated these rules and their consequences to children ${ }^{(26)}$ (for items, see Table 1). These items have also been used successfully in prior research ${ }^{(47)}$. Higher scores indicate more agreement with having, communicating and explaining rules; and the scale showed high internal reliability in the present sample (T1, $\alpha=0.84 ; \mathrm{T} 2, \alpha=0.85$ ).

Positive family meal practices. Five items from the Midwestern Prevention Project were averaged to create an overall score for frequency of positive family meal practices in the past $30 \mathrm{~d}^{(48,49)}$ (for items, see Table 1). Higher scores indicated more frequent use of positive family meal practices. Internal reliability was somewhat low (T1, $\alpha=0.64 ; \mathrm{T} 2, \alpha=0.61$ ).

Anhedonia. Anhedonia was included as a covariate in the statistical model. Anhedonia is a reduced or low ability to experience positive affect and is a subcomponent of depression ${ }^{(50)}$. Maternal depression may increase a child's risk for obesity and could confound the relationship with stress $^{(51,52)}$. Parent emotion and negative affectivity may affect the parent - child interaction during feeding of fruits and vegetables ${ }^{(53)}$, and adult anhedonia has been associated with quitting a weight-loss intervention programme, less weight lost, binge eating, uncontrolled eating and emotional eating ${ }^{(54,55)}$. Anhedonia has been shown to be inversely associated with walking and moderate and 
Table 1 Items for parent measures used in the present study

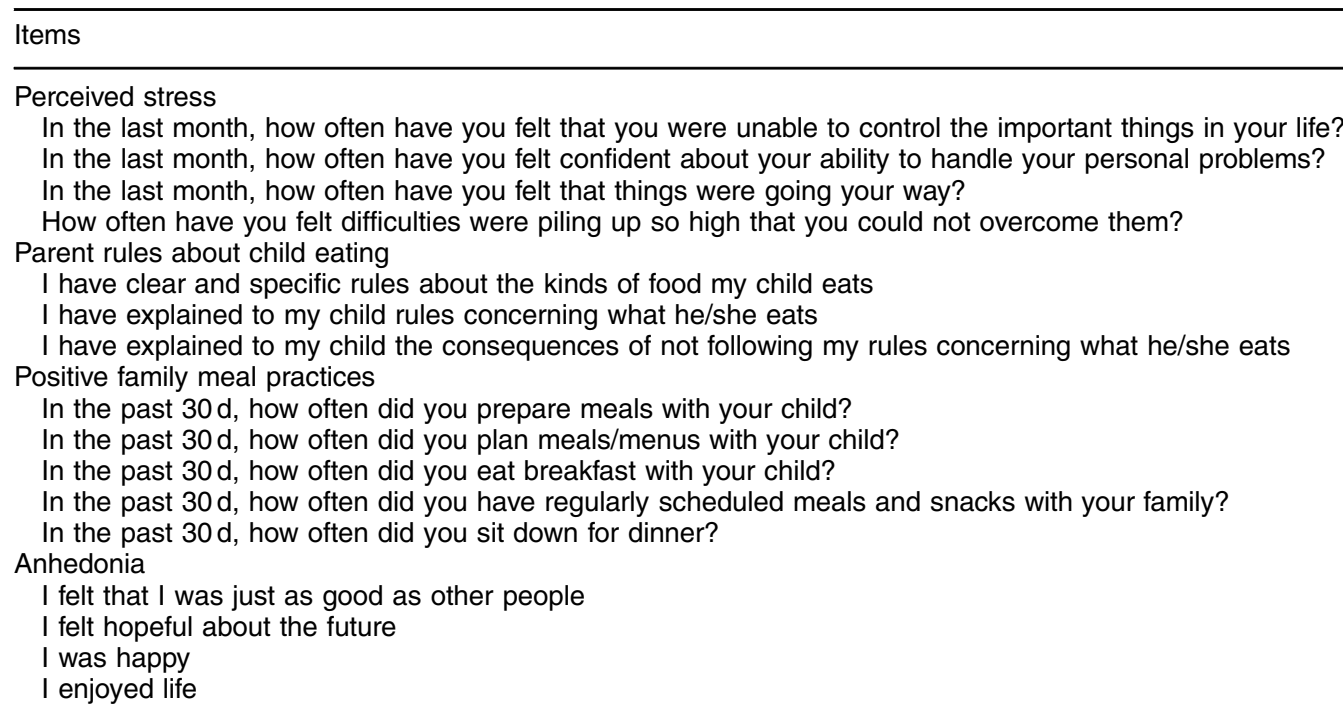

Response options for perceived stress: 1 = 'never', 2 = 'almost never', 3 = 'sometimes', 4 = 'fairly often', $5=$ 'very often'. Response options for parent rules about child eating: $1=$ 'strongly disagree', 2 ='disagree', 3 = 'neutral/mixed', 4 = 'agree', 5 = 'strongly agree'. Response options for positive family meal practices: $0=$ 'never', $1=$ 'rarely', $2=$ 'sometimes', $3=$ 'frequently', $4=$ 'always'. Response options for anhedonia: $1=$ 'rarely or none of the time' ( $0-1 \mathrm{~d}), 2=$ 'some or a little of the time' $(2-3 d), 3=$ 'occasionally or a moderate amount of the time' $(4-5 d), 4=$ 'most or all of the time' (6-7 d).

vigorous physical activity ${ }^{(50)}$. Anhedonia was measured using the four-item positive affect subscale of the Center for Epidemiologic Studies Depression scale ${ }^{(56)}$ (for items, see Table 1). Responses were reverse-coded and averaged for an overall score.

\section{Data analysis}

Composite scores, descriptive statistics and Pearson product - moment correlations were calculated and examined for outliers, skewness and kurtosis. Cross-sectional correlations were calculated between all variables at T1; and correlations were calculated between the predictor (i.e. parent stress) at $\mathrm{T} 1$, the hypothesized parenting practice mediators (i.e. rules and family meals) at T2 and the outcomes (i.e. child waist circumference, child added sugar intake) at T2. Mean differences in study variables between $\mathrm{T} 1$ and $\mathrm{T} 2$ were tested using paired $t$ tests. Dietary data were screened and cleaned as follows. Outliers of below $2092 \mathrm{~kJ} / \mathrm{d}(500 \mathrm{kcal} / \mathrm{d})$ and above $33471 \mathrm{~kJ} / \mathrm{d}$ $(8000 \mathrm{kcal} / \mathrm{d})$ were flagged as possible errors ${ }^{(57)}$. Raw distributions were examined for any entries greater than or less than 3 SD from the mean. Means, sD, skew and kurtosis for dietary variables were examined, and BMI was used to cross-validate in cases where children indicated consuming more than $12552 \mathrm{~kJ} / \mathrm{d}(3000 \mathrm{kcal} / \mathrm{d})$. Energy distributions were examined by age, ethnicity, BMI and socio-economic status.

For testing of the four study hypotheses, a path model was specified using Mplus ${ }^{(58)}$ with a Maximum Likelihood estimation with robust standard errors (i.e. 'MLR'), which is robust to violations of multivariate normality ${ }^{(59,60)}$. Criteria used to assess model fit were: goodness-of-fit $\chi^{2} P>0.05$; comparative fit index $(\mathrm{CFI}) \geq 0.95$; root-mean-square error of approximation (RMSEA) $\leq 0.05$ with the upper limit on the $90 \% \mathrm{CI}$ of $\leq 0 \cdot 10$; and standardized root-mean-square residual (SRMR) $\leq 0.08$ (and individual correlation residuals $\leq|0 \cdot 10|)^{(59)}$. Standardized estimates are reported in Fig. 1. The mediational model included two time points adjusted for demographic characteristics and the baseline level of each mediator ${ }^{(61)}$. Four indirect (i.e. mediated) effects of parent stress were modelled: (i) the indirect effect of parent stress leading to parent rules leading to child waist circumference; (ii) parent stress leading to family meal practices, leading to child waist circumference; (iii) parent stress leading to parent rules, leading to child consumption of added sugars; and (iv) parent stress leading to family meal practices, leading to child intake of added sugars. Each indirect effect was the product of the effect of parent stress on one mediator (e.g. parent rules; $a$ path) and the effect of that mediator on one outcome (e.g. child waist circumference; $b$ path) ${ }^{(62)}$. Demographics, parent anhedonia and study group (i.e. treatment $v$. control community) were screened and included as covariates in the final model because they demonstrated an association $(P<0 \cdot 10)$ with the outcome.

\section{Results}

\section{Sample characteristics}

Participant characteristics are shown in Table 2, including unadjusted means, SD, skewness, kurtosis, minimum and maximum. Parents in the study sample were mostly female (81\%), 39.2 (SD 6.00) years old, $70 \%$ graduated from college and $51 \%$ were Hispanic. The sample of children 


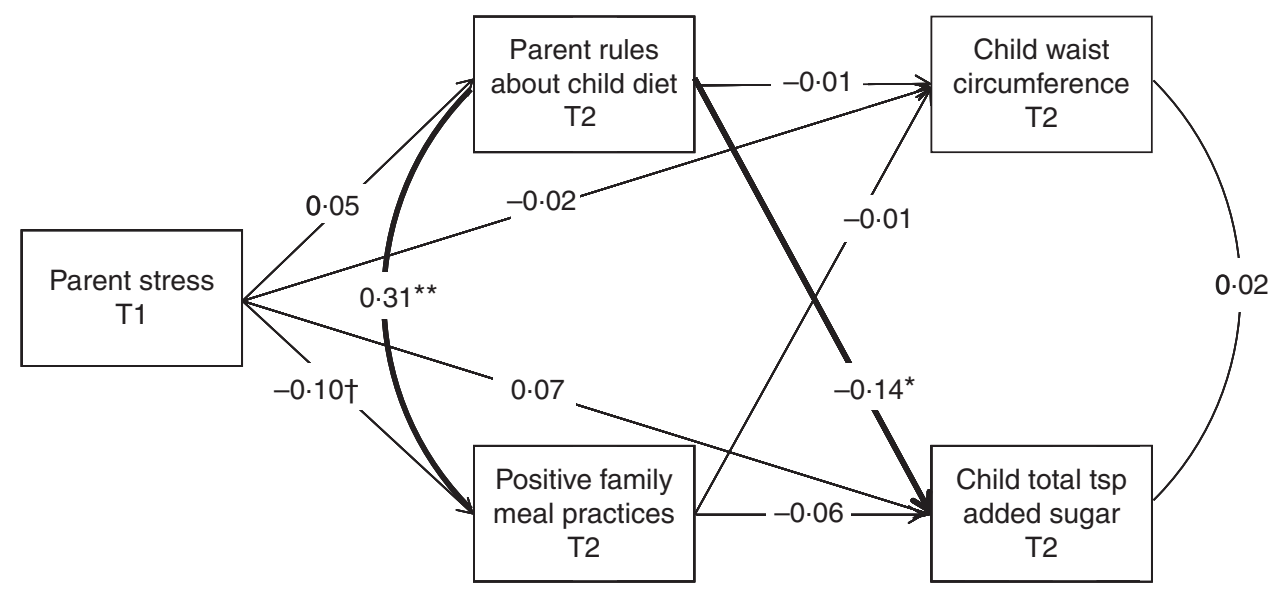

Fig. 1 Path model with parent stress at baseline (T1) predicting parenting practices and child waist circumference and added sugar intake one year later (T2) in a sample of Southern California adolescents. Model adjusts for child sex, age, ethnicity, free/reducedprice school lunch, group (treatment $v$. control), parent gender, parent anhedonia, and baseline levels of outcome and mediator variables. $\dagger P=0.06,{ }^{*} P<0.05,{ }^{* \star} P<0.01 . X^{2}(12)=19.00, P=0.09$; comparative fit index $=0.99$; root-mean-square error of approximation $=0.04(95 \% \mathrm{Cl} 0.00,0.07)$; standardized root-mean-square residual $=0.02 ; n 385$

Table 2 Descriptive statistics for study variables

\begin{tabular}{|c|c|c|c|c|c|c|c|}
\hline Description & $n$ & Mean & SD & Skewness & Kurtosis & Minimum & Maximum \\
\hline Parent perceived stress $\mathrm{T} 1$ & 597 & 9.20 & $2 \cdot 81$ & 0.18 & -0.21 & 4.00 & $18 \cdot 00$ \\
\hline Parent perceived stress T2 & 437 & $9 \cdot 38$ & $2 \cdot 85$ & 0.25 & -0.11 & 4.00 & 19.00 \\
\hline Parent rules about child diet T1 & 596 & 3.63 & 0.84 & -0.47 & 0.38 & 1.00 & 5.00 \\
\hline Parent rules about child diet T2 & 448 & 3.60 & 0.85 & -0.57 & 0.50 & 1.00 & 5.00 \\
\hline Positive family meal practices $\mathrm{T} 1$ & 596 & $2 \cdot 34$ & 0.67 & -0.19 & 0.24 & 0.00 & 4.00 \\
\hline Positive family meal practices $\mathrm{T} 2$ & 437 & $2 \cdot 38$ & 0.63 & -0.10 & 0.21 & 0.40 & 4.00 \\
\hline Child waist circumference T1 & 595 & 74.86 & 12.55 & 0.86 & 1.05 & $51 \cdot 10$ & $140 \cdot 60$ \\
\hline Child waist circumference T2 & 457 & $77 \cdot 78$ & 12.91 & $1 \cdot 10$ & $2 \cdot 33$ & 52.50 & $146 \cdot 55$ \\
\hline Child dietary added sugar tsp $\mathrm{T} 1$ & 539 & $6 \cdot 20$ & 3.67 & 1.54 & 4.07 & 0.31 & $25 \cdot 20$ \\
\hline Child dietary added sugar tsp T2 & 398 & $6 \cdot 10$ & 3.77 & $2 \cdot 11$ & 7.96 & 0.45 & 28.24 \\
\hline Child age (years) & 595 & 11.29 & 1.53 & 0.20 & -0.68 & $7 \cdot 75$ & $15 \cdot 01$ \\
\hline Parent age (years) & 596 & $39 \cdot 20$ & 6.00 & 0.35 & 0.13 & 23.00 & $62 \cdot 00$ \\
\hline Parent anhedonia T1 & 594 & 1.55 & 0.64 & 1.25 & 1.05 & 1.00 & 4.00 \\
\hline
\end{tabular}

T1, time 1 (baseline); T2, time 2 (one year later).

Parent perceived stress, range: 4 (low) to 20 (high). Parent rules about child diet, range: 1 (strongly disagree) to 5 (strongly agree). Frequency of positive family meal practices, range: 0 (never) to 4 (always). Parent anhedonia, range: 1 (rarely or none of the time) to 4 (most or all of the time).

was $53 \%$ male; 11.3 (sD 1.53) years old; $42 \%$ Hispanic, $26 \%$ Caucasian, 10\% Asian, 6\% African-American and $17 \%$ Other; $31 \%$ received free school lunches.

\section{Child adiposity and diet}

Child waist circumference at T1 was 74.86 (SD 12.55) cm (range $51 \cdot 10-140 \cdot 60 \mathrm{~cm}$ ) and at T2 was $77.78(\mathrm{sD} 12 \cdot 91) \mathrm{cm}$ (range $52.50-146.55 \mathrm{~cm}$ ); these differed significantly, as is expected for normal growth $(\Delta=\mathrm{T} 2-\mathrm{T} 1=2.97$ (SD 7.49) $\mathrm{cm} ; t=8.47, P<0.0001)$. The sample had a higher rate of obesity at T2 (9.2\%) compared with T1 (6.2\%; McNemar's $S(1)=7.54, P<0 \cdot 01)$, defining obesity as age- and genderadjusted waist circumferences above the 95th percentile $^{(63)}$. All variables had relatively normal distributions except for child consumption of added sugars at T2, which had skewness $>2$ and kurtosis $>7$; values this high have been found to be problematic in simulation models ${ }^{(64)}$, although others recommend higher cut-offs, such as skewness $>3$ and kurtosis $>8$ or $10^{(59)}$ (Table 2). Children consumed a little over 6 teaspoons of added sugar on average per $4184 \mathrm{~kJ}$ (1000 kcal) per d at T1 (SD $3.67 \mathrm{tsp}$; Table 2). The change in child consumption of added sugars from $\mathrm{T} 1$ to $\mathrm{T} 2$ was not significant $(\Delta=\mathrm{T} 2-\mathrm{T} 1=$ -0.07 (sD 4.47) tsp; $t=-0.31, P>0.05$ ).

\section{Parent stress and parenting practices}

At T1, the average parent stress score was between the 'almost never' and 'sometimes' within the last month (mean $9 \cdot 20$ (SD 2.81); range 4-18; $\alpha=0.73$ ); having positive family meal practices was between 'sometimes' and 'frequently' (mean 2.34 (SD 0.67); range 0-4); and the average parent agreement about having rules about children's diet was between 'neutral/mixed' and 'agree' (mean 3.63 (sD 0.84); range $1-5$; see Table 2$)$. Parent stress $(\Delta=\mathrm{T} 2-\mathrm{T} 1=0 \cdot 17$ (SD 2.59), $t=1.37)$, parent rules $(\Delta=\mathrm{T} 2-\mathrm{T} 1=-0.07$ (SD 0.81), $t=-1.75)$ and positive family meal practices $(\Delta=\mathrm{T} 2-\mathrm{T} 1=0.02$ (SD 1.65), $t=0.72)$ did not change significantly between time points $(P>0 \cdot 05)$. 
Correlations among parent stress, parenting practices, child consumption of added sugars and child waist circumference

Table 3 shows bivariate cross-sectional correlations among study variables at T1, and correlations between parent stress at T1 and parent rules, family meal practices, child added sugar intake and child waist circumference at T2. In line with Hypothesis 1, parents with higher stress at T1 had fewer rules about child eating $(r=-0 \cdot 11, P<0 \cdot 01)$, fewer positive family meal practices $(r=-0.09, P<0.05)$ and children with larger waist circumferences at T1 $(r=0.09, P<0.05)$ but not T2 ( $r=0.02$, NS; see Table 3 ). At T1, higher parent rules about diet were correlated with greater positive family meal practices $(r=0.31, P<0.001)$ and fewer teaspoons of added sugars at T1 $(r=-0 \cdot 16, P<0 \cdot 01)$. However, child teaspoons of added sugars at T1 were not significantly correlated with child waist circumference at T1 $(r=-0 \cdot 05)$ or T2 $(r=-0 \cdot 01)$.

\section{Model results for direct and indirect effects of perceived stress on parenting practices and change in child waist circumference and added sugar intake}

The path model is shown in Fig. 1. The model fit the data well except for the $\chi^{2}$ statistic, which was expected to be large due to the high number of observations: $\chi^{2}(12)=19 \cdot 00, P=0.09 ; \mathrm{CFI}=0.99 ; \mathrm{RMSEA}=0.04(95 \% \mathrm{CI}$ $0 \cdot 00,0 \cdot 07) ; \mathrm{SRMR}=0 \cdot 02 ; n 385$.

Contrary to Hypothesis 2, parent stress at T1 did not significantly predict child waist circumference $(\beta=-0 \cdot 02$, NS) or child consumption of added sugars $(\beta=0 \cdot 07$, NS) at T2. Contrary to Hypothesis 3, the indirect effect of parent stress on child waist circumference at $\mathrm{T} 2$ was not significant through parent rules (indirect effect $=-0.001$ (SE 0.005), $P=0 \cdot 81$ ) or positive family meal practices (indirect effect $=$ 0.005 (se 0.012), $P=0.68$ ). Contrary to Hypothesis 4, the indirect effect of parent stress on child added sugar intake at T2 was not significant through parent rules (indirect effect $=-0.009$ (sE 0.014), $P=0.51$ ) or through positive family meal practices (indirect effect $=0.008$ (se 0.008), $P=0.33$ ). However, parent rules at T2 were significantly associated with lower child consumption of added sugars at T2 $(\beta=-0 \cdot 14, P=0 \cdot 02)$ and positive family meal practices at T2 $(r=0.31, P<0 \cdot 0001)$. A post boc median split (median $=$ 3.67) of parents into high $v$. low rules revealed that children of 'high rules' parents consumed 5.43 teaspoons of added sugar per $4184 \mathrm{~kJ}(1000 \mathrm{kcal})$ per d on average compared with 'low rules' parents (6.59 tsp/4184 kJ ( $1000 \mathrm{kcal})$ per d), which differed significantly (Satterthwaite $t$ test for unequal variances $t(395 \cdot 12)=3 \cdot 26, P<0 \cdot 01)$.

\section{Discussion}

Higher parent stress was cross-sectionally correlated with fewer parent rules, fewer positive family meal practices and higher child waist circumference. However, in

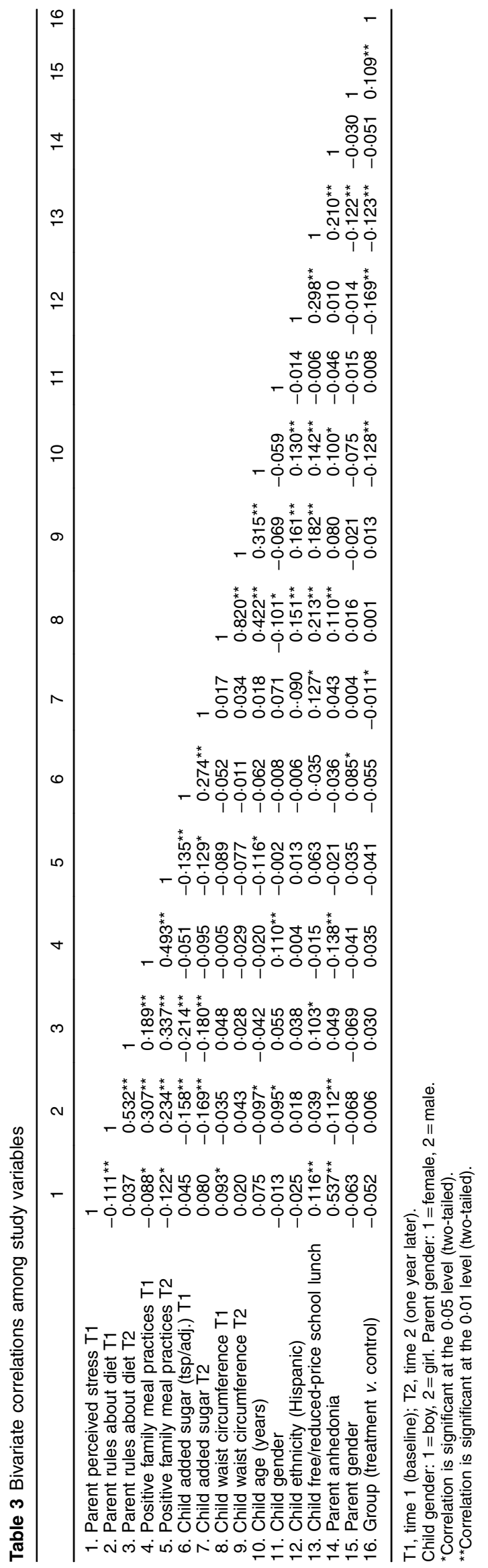


contrast to hypotheses, the longitudinal model results indicated that higher parent stress did not indirectly affect child waist circumference or added sugar intake through parent rules or family meal practices. Yet, parent rules about child diet were associated with lower child consumption of added sugars. While these findings support a connection between parents' perceived stress and weightrelated parenting practices, prospective effects on child obesity risk were not observed. Future research with larger samples is needed to test multiple weight-related parenting practices as well as potential feedback loops, such as child obesity leading to parent stress or to changes in parenting practices.

The present study addressed two gaps in the literature. First, the effect of subjective, perceived stress on parenting practices and child obesity risk was examined in contrast to previous work which has focused mainly on external, 'objective' stressors ${ }^{(14,65)}$. Previous research has shown that circumstances such as poverty or experiencing challenging situations like divorce may reflect difficult family settings that undergird health disparities in obesity. Thus, it was important to investigate whether perceived stress, which would be assumed to co-vary with stressors, showed the same relationship. Previous work indicated an association between parent stress and physical activity parenting ${ }^{(14)}$, supporting the current finding with dietrelated parenting practices. Yet previous studies have not found a significant link between perceived stress and child $\mathrm{BMI}^{(13,14)}$. Instead of BMI, the current study examined effects on child waist circumference, finding a significant correlation that became non-significant after accounting for other strong influences such as free school lunch status and ethnicity. The fact that the null findings regarding child obesity risk are consistent with two other studies suggests that objective stressors and perceived stress operate differently on child obesity risk. Stress that parents experienced in the last month (as measured by the Cohen Perceived Stress scale ${ }^{(44)}$ ) may dissipate too quickly to contribute to children's obesity risk over one year, but whether parent perceived stress that accumulates chronically over that year could affect weight-related practices during the year, ultimately heightening child obesity risk, remains unknown. Overall, findings indicate that parent perceived stress tends to be related to having heavier children but, alone, does not increase child obesity risk in the way that enduring objective stressors does.

Second, the study sought to elucidate the processes by which perceived parent stress could affect child added sugar intake, specifically through fewer parent rules or fewer positive family meal practices. In this sample, parent perceived stress was correlated with fewer parent rules and positive family meal practices, but the effects were small and became non-significant after adjusting for other influences such as child age, gender and parent anhedonia. The timing of measures may be an explanation for the lack of significant findings. The measure of perceived stress was retrospective over the past month, but the measures of parenting practices were static indicators of whether parents had any rules or tended to have regular meals. If the effects of perceived stress operate on a much more proximal scale - if a particularly stressful day increases a mother's likelihood of purchasing high-sugar convenience foods that day - the current study would not necessarily capture that link. Thus, studies are needed to assess within-daily effects of perceived and objective stressors on child weight-related behaviours and accumulated obesity risk over time. In sum, parent perceived stress may have a small effect on weight-related parenting practices but findings were inconclusive, and future research with larger sample sizes and more proximate measures is needed.

Child waist circumference in this sample at baseline was slightly higher than national 2007-2010 estimates for 11-year-old males $(71.9 \mathrm{~cm})$ and females $(73.2 \mathrm{~cm})^{(63)}$, which may align with the fact that the sample was deliberately drawn to oversample Hispanic families, which have higher rates of child obesity ${ }^{(66)}$. Children consumed about 6 teaspoons of added sugar per $4184 \mathrm{~kJ}(1000 \mathrm{kcal})$ per $\mathrm{d}$; this is lower than one estimate of nationwide intake, 9.63 teaspoons per $4184 \mathrm{~kJ}$ (1000 kcal) per d,* but the Block Kids' Dietary Screener may underestimate added sugar $^{(41,67)}$. Additionally, the American Heart Association recommends less than or equal to $\approx 6$ teaspoons of added sugar daily, which is $3 \cdot 2$ teaspoons per $4184 \mathrm{~kJ}$ ( $1000 \mathrm{kcal})$ at a daily energy intake of $7950 \mathrm{~kJ}$ (1900 kcal; the middle of the range recommended for children aged 9-13 years ${ }^{(68,69)}$. While previous studies indicate an association between higher parent rules and lower child sugar consumption $^{(18,20,21)}$, cross-sectional studies cannot untangle whether parents create rules because their children consume too many added sugars or whether parent rules cause children to consume less sugar. The current longitudinal findings shed some light on this question, suggesting that parent rules could lead to lower sugar consumption, if not lower abdominal obesity, over time. Because measures of parent rules and sugar consumption were measured at two time points, baseline levels could be controlled for in the analysis, which is an advantage over cross-sectional designs. However, this interpretation rests on the assumption that the magnitude of the effect of parent rules on child consumption of added sugars between T1 and T2 in the present study would be the same as the magnitude between T2 and a (hypothetical) $\mathrm{T} 3^{(61)}$. Thus, a future study with three time points would strengthen this interpretation.

Finally, the present study contributes to the discussion on the effects of parent dietary rules on child diet and

\footnotetext{
* In 2005-2008, the Centers for Disease Control and Prevention estimated that school-aged children consumed an average of $1335 \mathrm{~kJ}$ (319kcal) of added sugar daily ((girls $293+$ boys 345$) / 2=319 \mathrm{kcal}$ ), or about 19 teaspoons. The mean percentage of total energy from added sugar (girls $15.7 \%$; boys $16.6 \%$ ) indicates that total daily energy intake was $8251 \mathrm{~kJ}$ (1972 kcal) on average ((girls $1866+$ boys 2078$) / 2=1972 \mathrm{kcal})$, indicating consumption of 9.63 teaspoons per $4184 \mathrm{~kJ}(1000 \mathrm{kcal})((19 \times 1000) / 1972)$.
} 
weight where prior research reveals counterintuitive, conflicting results. While rules have been associated with lower consumption of added sugar and sugary beverages, dietary restrictions are also associated with child weight gain and overweight ${ }^{(19,70)}$. This is counterintuitive because lower sugar intake would be expected to lead to lower obesity risk, not higher. Restrictive rules could curb present consumption of sweets but increase taste preference for sweetness ${ }^{(20)}$ or hinder children's ability to develop self-regulatory control over eating behaviour, at least in girls ${ }^{(71)}$. One study has found that parent rules lower the risk of overweight ${ }^{(26)}$. One reason for the discrepancies is different measures of parent rules. The current study assessed rules that were clearly explained to children along with the consequences of not following those rules, but specific motivations or intentions behind those rules were not measured. In contrast, the bulk of experimental and longitudinal evidence implicating maternal restriction of palatable foods in child weight gain tends to measure specific cognitions relating to a perceived need to control the child's impulses and access to food (e.g. ${ }^{(70,72)}$ ). Results from the current study support previous findings that rules unrelated to these specific types of cognitions may lead to lower sugar consumption but not necessarily affect obesity risk.

\section{Limitations}

The current study assessed the weight-related parenting practices of rules about eating and family meal practices, but other practices not measured here may be correlated with parent stress or have stronger effects on child obesity risk. For example, understanding specific rules about how or what the child eats may help uncover which rules actually lead to changes in child obesity risk, suggesting a moderating, rather than mediating, effect of parenting practices. The positive family meal practices indicator had low internal reliability in this sample, suggesting that an alternative measure may better capture these practices in similar samples. Physical activity parenting was not measured in the current study but could be affected by parent stress, accumulating into changes in waist circumference over time. Other facets of weight-related parenting, such as restrictive feeding practices, may also interact with parent perceived stress to influence child weight. A second limitation is the time frame: two waves of data measured over one year. In this type of model, the significance test for the mediational effect of $\mathrm{M}$ on $\mathrm{Y}$ (the $c$ path) can only indicate partial mediation, not full mediation. However, our study did not find evidence of mediation (i.e. the indirect effects were non-significant), so there was no question of whether M fully, or only partially, explained the relationship between $\mathrm{X}$ and $\mathrm{Y}^{(61)}$. Additionally, effects of parent stress on parenting practices and child health behaviour could occur within very short time frames - perhaps even within the day - while changes in waist circumference develop over a much longer time. Yet, the time frame of the present study would mask those effects. Third, while the present study contributes to our understanding of perceived stress, objective stressors were not studied. Hence, direct comparisons between effects of perceived $v$. objective stressors - or how they interact - could not be investigated. Further research is needed that contextualizes perceived stress within the context of objective stressors and examines potential effects on parenting practices, child dietary intake and obesity risk. Fourth, the study population was not nationally representative, and findings may have been influenced by more highly educated parents or features of the Southern California environment. Finally, methodological limitations such as a lack of power may have contributed to the non-significant overall model, suggesting that studies with larger samples or repeated measures on within-daily effects may be needed to understand these relationships.

\section{Conclusion}

The times in life when parents perceive themselves to have inadequate control over their lives and to feel worried - when they experience perceived stress - may not relate to the parenting practices they engage in later or their children's subsequent obesity risk. Parent perceived stress does not seem to impact child obesity risk the way parents' external stressors, such as poverty, do. These findings suggest that child obesity prevention efforts are likely to have a greater impact if they alleviate challenging circumstances in which families find themselves rather than decreasing the experience of perceived stress per se. Further, these findings support efforts to educate and support parents in clearly communicating rules for their children's diets as a possible means of decreasing consumption of added sugars to levels more aligned with recommendations. Future longitudinal research is needed to examine effects of specific rules and other parenting practices on child dietary intake, and other potential mediating influences, to explain the link between external stressors that parents experience and child obesity risk.

\section{Acknowledgements}

Financial support: This work was supported by a National Institutes of Health Cancer Control and Epidemiology Research Training Grant (Principal Investigator M.A.P., grant number 5 T32 CA 009492); and the American Cancer Society (Principal Investigator G.F.D., grant number \#118283-MRSGT-10-012-01-CPPB). The funders had no role in the design, analysis or writing of this article. Conflict of interest: None to report. Authorship: Authors' contributions were as follows. E.T.S.: conceptualization of the research question, data analysis, writing of the manuscript. G.F.D.: study design, conceptualization of the research question, data analytic planning, editing of the manuscript. C.-P.C.: data analytic planning and supervision, editing of the 
manuscript. A.M.L.: conceptualization of the research question and analysis plan, editing of the manuscript. R.B.: conceptualization of the research question and analysis plan, editing of the manuscript. M.A.P.: study design, supervision of carrying out the study, conceptualization of the research question, editing of the manuscript. Ethics of human subject participation: This study was conducted according to the guidelines laid down in the Declaration of Helsinki and all procedures involving human subjects were approved by the University of Southern California Institutional Review Board. Written informed consent or child assent was obtained from all subjects.

\section{References}

1. McPhie S, Skouteris H, McCabe M et al. (2011) Maternal correlates of preschool child eating behaviours and body mass index: a cross-sectional study. Int J Pediatr Obes 6, 476-480.

2. Gundersen C, Lohman BJ, Garasky S et al. (2008) Food security, maternal stressors, and overweight among lowincome US children: results from the National Health and Nutrition Examination Survey (1999-2002). Pediatrics 122, e529-e540.

3. Kozyrskyj AL, Zeng Y, Colman I et al. (2011) Maternal distress in early life predicts the waist-to-hip ratio in schoolchildren. J Dev Orig Health Dis 2, 72-80.

4. Lohman BJ, Stewart S, Gundersen C et al. (2009) Adolescent overweight and obesity: links to food insecurity and individual, maternal, and family stressors. J Adolesc Health 45, 230-237.

5. Stenhammar C, Olsson GM, Bahmanyar S et al. (2010) Family stress and BMI in young children. Acta Paediatr 99, 1205-1212.

6. Suglia SF, Duarte CS, Chambers EC et al. (2012) Cumulative social risk and obesity in early childhood. Pediatrics 129, e1173-e1179.

7. Tate EB, Wood W, Liao Y et al. (2015) Do stressed mothers have heavier children? A meta-analysis on the relationship between maternal stress and child body mass index. Obes Rev 16, 351-361.

8. Lundahl A, Nelson TD, Van Dyk TR et al. (2013) Psychosocial stressors and health behaviors: examining sleep, sedentary behaviors, and physical activity in a low-income pediatric sample. Clin Pediatr 52, 721-729.

9. Park H \& Walton-Moss B (2012) Parenting style, parenting stress, and children's health-related behaviors. J Dev Behav Pediatr 33, 495-503.

10. Conger KJ, Rueter MA \& Conger RD (2000) The role of economic pressure in the lives of parents and their adolescents: the Family Stress Model. In Negotiating Adolescence in Times of Social Change, pp. 201-223 [LJ Crockett and RK Silberiesen, editors]. Cambridge: Cambridge University Press.

11. McCurdy K, Gorman KS \& Metallinos-Katsaras E (2010) From poverty to food insecurity and child overweight: a family stress approach. Child Dev Perspect 4, 144-151.

12. Cohen S, Kessler RC \& Underwood Gordon L (editors) (1995) Strategies for measuring stress in studies of psychiatric and physical disorders. In Measuring Stress: A Guide for Health and Social Scientists, pp. 3-26. New York: Oxford University Press.

13. Parks EP, Kumanyika S, Moore RH et al. (2012) Influence of stress in parents on child obesity and related behaviors. Pediatrics 130, e1096-e1104.

14. Walton K, Simpson JR, Darlington G et al. (2014) Parenting stress: a cross-sectional analysis of associations with childhood obesity, physical activity, and TV viewing. BMC Pediatr 14, 244.

15. Hurley KM, Black MM, Papas MA et al. (2008) Maternal symptoms of stress, depression, and anxiety are related to nonresponsive feeding styles in a statewide sample of WIC participants. J Nutr 138, 799-805.

16. Mitchell S, Brennan L, Hayes L et al. (2009) Maternal psychosocial predictors of controlling parental feeding styles and practices. Appetite 53, 384-389.

17. Eisenberg CM, Ayala GX, Crespo NC et al. (2012) Examining multiple parenting behaviors on young children's dietary fat consumption. J Nutr Educ Behav 44, 302-309.

18. Gubbels JS, Kremers SPJ, Stafleu A et al. (2009) Diet-related restrictive parenting practices. Impact on dietary intake of 2 -year-old children and interactions with child characteristics. Appetite 52, 423-429.

19. Hauser SI, Economos CD, Nelson ME et al. (2014) Household and family factors related to weight status in first through third graders: a cross-sectional study in Eastern Massachusetts. BMC Pediatr 14, 167.

20. Liem DG, Mars M \& De Graaf C (2004) Sweet preferences and sugar consumption of 4- and 5-year-old children: role of parents. Appetite 43, 235-245.

21. Verzeletti C, Maes L, Santinello M et al. (2010) Soft drink consumption in adolescence: associations with foodrelated lifestyles and family rules in Belgium Flanders and the Veneto Region of Italy. Eur J Public Health 20, 312-317.

22. Rodriguez LA, Madsen KA, Cotterman C et al. (2016) Added sugar intake and metabolic syndrome in US adolescents: cross-sectional analysis of the National Health and Nutrition Examination Survey 2005-2012. Public Health Nutr 19, 2424-2434.

23. Della Torre SB, Keller A, Depeyre JL et al. (2016) Sugarsweetened beverages and obesity risk in children and adolescents: a systematic analysis on how methodological quality may influence conclusions. I Acad Nutr Diet 116, 638-659.

24. Keller A \& Della Torre SB (2015) Sugar-sweetened beverages and obesity among children and adolescents: a review of systematic literature reviews. Child Obes $\mathbf{1 1}$, 338-346.

25. Gubbels JS, Kremers SPJ, Stafleu A et al. (2011) Association between parenting practices and children's dietary intake, activity behavior and development of body mass index: the KOALA Birth Cohort Study. Int J Behav Nutr Phys Act 8, 18 .

26. Lytle LA, Hearst MO, Fulkerson J et al. (2011) Examining the relationships between family meal practices, family stressors, and the weight of youth in the family. Ann Behav Med 41, 353-362.

27. Li C, Ford ES, Mokdad AH et al. (2006) Recent trends in waist circumference and waist-height ratio among US children and adolescents. Pediatrics 118, e1390-e1398.

28. Spolidoro JV, Pitrez ML, Vargas LT et al. (2013) Waist circumference in children and adolescents correlate with metabolic syndrome and fat deposits in young adults. Clin Nutr 32, 93-97.

29. Shearrer GE, Daniels MJ, Toledo-Corral CM et al. (2016) Associations among sugar sweetened beverage intake, visceral fat, and cortisol awakening response in minority youth. Physiol Behav 167, 188-193.

30. Bigornia SJ, LaValley MP, Noel SE et al. (2015) Sugarsweetened beverage consumption and central and total adiposity in older children: a prospective study accounting for dietary reporting errors. Public Health Nutr 18, 1155-1163.

31. Pentz MA, Dunton G, Huh J et al. (2010) Effects of living in a smart growth community on social and environmental connectivity, and physical activity: the Healthy Places Trial. Obesity 18, 558.

32. Pentz MA, Dunton G, Wolch J et al. (2010) Design and methods of the healthy places trial: a study of the effects of smart growth planning principles on family obesity prevention. Ann Behav Med 39, 42. 
33. Almanza E, Jerrett M, Dunton G et al. (2012) A study of community design, greenness, and physical activity in children using satellite, GPS and accelerometer data. Health Place 18, 46-54

34. Dunton GF, Intille SS, Wolch J et al. (2012) Investigating the impact of a smart growth community on the contexts of children's physical activity using ecological momentary assessment. Health Place 18, 76-84.

35. Dunton GF, Liao Y, Kawabata K et al. (2012) Momentary assessment of adults' physical activity and sedentary behavior: feasibility and validity. Front Psychol 3, 260.

36. Perez-Pastor EM, Metcalf BS, Hosking J et al. (2009) Assortative weight gain in mother - daughter and father son pairs: an emerging source of childhood obesity. Longitudinal study of trios (EarlyBird 43). Int J Obes (Lond) 33, $727-735$.

37. Cullen KW, Baranowski T, Rittenberry L et al. (2000) Socialenvironmental influences on children's diets: results from focus groups with African-, Euro- and Mexican-American children and their parents. Health Educ Res 15, 581-590.

38. US Department of Agriculture (2009) Child Nutrition Programs: Income Eligibility Guidelines (July 1, 2009-June 30, 2010). https://www.gpo.gov/fdsys/pkg/FR-2009-03-27/pdf/ E9-6806.pdf (accessed August 2017).

39. Moens E, Braet C, Bosnians G et al. (2009) Unfavourable family characteristics and their associations with childhood obesity: a cross-sectional study. Eur Eat Disord Rev 17, 315-323.

40. Santiago S, Zazpe I, Cuervo M et al. (2012) Perinatal and parental determinants of childhood overweight in 6-12 years old children. Nutr Hosp 27, 599-605.

41. Hunsberger M, O'Malley J, Block T et al. (2012) Relative validation of Block Kids Food Screener for dietary assessment in children and adolescents. Matern Child Nutr 11, 260-270.

42. Comstock EM, St Pierre RG \& Mackiernan YD (1981) Measuring individual plate waste in school lunches. Visual estimation and children's ratings vs. actual weighing of plate waste. J Am Diet Assoc 79, 290-296.

43. Bowman SA, Friday JE \& Moshfegh AJ (2008) MyPyramid Equivalents Database, 2.0 for USDA Survey Foods, 20032004: Documentation and User Guide. Beltsville, MD: Food Surveys Research Group, Beltsville Human Nutrition Research Center, Agricultural Research Service, US Department of Agriculture.

44. Cohen S, Kamarck T \& Mermelstein R (1983) A global measure of perceived stress. J Health Soc Behav 24, 385-396.

45. Lee E-H (2012) Review of the psychometric evidence of the Perceived Stress Scale. Asian Nurs Res 6, 121-127.

46. Nast I, Bolten M, Meinlschmidt G et al. (2013) How to measure prenatal stress? A systematic review of psychometric instruments to assess psychosocial stress during pregnancy. Paediatr Perinat Epidemiol 27, 313-322.

47. Hearst MO, Sevcik S, Fulkerson JA et al. (2012) Stressed out and overcommitted! The relationships between time demands and family rules and parents' and their child's weight status. Health Educ Behav 39, 446-454.

48. Gattshall ML, Shoup JA, Marshall JA et al. (2008) Validation of a survey instrument to assess home environments for physical activity and healthy eating in overweight children. Int J Behav Nutr Phys Act 5, 3.

49. Pentz MA, Mihalic SF \& Grotpeter JK (1997) The Midwestern Prevention Project. Boulder, CO: Center for the Study and Prevention of Violence, Institute of Behavioral Science, University of Colorado.

50. Leventhal AM (2012) Relations between anhedonia and physical activity. Am J Health Behav 36, 860-872.

51. Ramasubramanian L, Lane S \& Rahman A (2013) The association between maternal serious psychological distress and child obesity at 3 years: a cross-sectional analysis of the
UK Millennium Cohort Data. Child Care Health Dev 39, 134-140.

52. Topham GL, Page MC, Hubbs-Tait L et al. (2010) Maternal depression and socio-economic status moderate the parenting style/child obesity association. Public Health Nutr 13, 1237-1244

53. Hughes SO, Power TG, Fisher JO et al. (2005) Revisiting a neglected construct: parenting styles in a child-feeding context. Appetite 44, 83-92.

54. Keranen AM, Rasinaho E, Hakko H et al. (2010) Eating behavior in obese and overweight persons with and without anhedonia. Appetite 55, 726-729.

55. Komulainen T, Keranen AM, Rasinaho E et al. (2011) Quitting a weight loss program is associated with anhedonia: preliminary findings of the Lifestyle Intervention Treatment Evaluation Study in northern Finland. Int $J$ Circumpolar Health 70, 72-78.

56. Radloff LS (1991) The use of the center for epidemiologic studies depression scale in adolescents and young-adults. I Youth Adolesc 20, 149-166.

57. Willett W (2012) Nutritional Epidemiology, 3rd ed. New York: Oxford University Press.

58. Muthén LK \& Muthén BO (1998-2011) Mplus User's Guide, 6th ed. Los Angeles, CA: Muthén \& Muthén.

59. Kline RB (2011) Principles and Practice of Structural Equation Modeling. New York: Guilford Press.

60. Muthén BO \& Muthén LK (2012) Mplus Version 7: User's Guide. Los Angeles, CA: Muthén \& Muthén.

61. Cole DA \& Maxwell SE (2003) Testing mediational models with longitudinal data: questions and tips in the use of structural equation modeling. J Abnorm Psychol 112, 558-577.

62. Preacher KJ \& Hayes AF (2008) Asymptotic and resampling strategies for assessing and comparing indirect effects in multiple mediator models. Behav Res Methods 40, 879-891.

63. Fryar GU, Carroll MD \& Ogden CL (2012) Prevalence of obesity among children and adolescents: United States, trends 1963-1965 through 2009-2010. National Center for Health Statistics, Health E-Stats. https://www.cdc.gov/nchs/ data/hestat/obesity_child_09_10/obesity_child_09_10.pdf (accessed September 2017).

64. Curran PJ, West SG \& Finch JF (1996) The robustness of test statistics to nonnormality and specification error in confirmatory factor analysis. Psychol Methods 1, 16-29.

65. Bauer KW, Hearst MO, Escoto K et al. (2012) Parental employment and work - family stress: associations with family food environments. Soc Sci Med 75, 496-504.

66. Ogden CL, Carroll MD, Kit BK et al. (2012) Prevalence of obesity and trends in body mass index among US children and adolescents, 1999-2010. JAMA 307, 483-490.

67. Ervin RB, Kit BK, Carroll MD et al. (2012) Consumption of added sugar among US children and adolescents, 20052008. NCHS Data Brief issue 87, 1-8; available at http:// www.cdc.gov/nchs/data/databriefs/db87.pdf

68. US Department of Health and Human Services (2010) Parent Tips: Calories Needed Each Day. https://www.nhlbi.nih. gov/health/educational/wecan/downloads/calreqtips.pdf (accessed January 2017).

69. Vos MB, Kaar JL, Welsh JA et al. (2016) Added sugars and cardiovascular disease risk in children. Circulation 135, e1017-e1034.

70. Clark HR, Goyder E, Bissell P et al. (2007) How do parents' child-feeding behaviours influence child weight? Implications for childhood obesity policy. J Public Health 29, 132-141.

71. Birch LL \& Fisher JO (2000) Mothers' child-feeding practices influence daughters' eating and weight. Am J Clin Nutr $\mathbf{7 1}$, 1054-1061.

72. Birch LL, Fisher JO, Grimm-Thomas K et al. (2001) Confirmatory factor analysis of the Child Feeding Questionnaire: a measure of parental attitudes, beliefs and practices about child feeding and obesity proneness. Appetite 36, 201-210. 